\title{
APPROCHE MULTI-ÉCHELLES DES RELATIONS ENTRE LES CARACTÉRISTIQUES D'HABITAT ET LES POPULATIONS DE TRUITE COMMUNE (SALMO TRUTTA L.) DANS LES PYRÉNÉES CENTRALES.
}

\author{
P. BARAN (1), M. DeLACOSTE (1), G. POIZAT (2), J.M. LASCAUX (1), \\ S. LEK (3), A. BELAUD (1).
}

(1) Laboratoire d'Ingénierie Agronomique, Equipe Environnement Aquatique et Aquaculture, ENSAT, 145 av. de Muret, 31076 Toulouse Cedex, France.

(2) Laboratoire d'Ecologie des Systèmes Fluviaux. URA CNRS 1451, Equipe DESMID, 1 rue Parmentier, 13200 Arles, France.

(3) Laboratoire de Biologie Quantitative, Université Paul Sabatier, 118 Route de Narbonne, 31062 Toulouse Cedex, France.

\section{RÉSUMÉ}

L'influence des échelles d'espace du tronçon, de la séquence et du faciès d'écoulement sur le taux d'occupation par les truites communes (Salmo trutta L.) de l'habitat physique défini par la méthode des microhabitats a été étudiée sur 264 faciès d'écoulement appartenant à 15 rivières des Pyrénées centrales. L'utilisation d'un modèle d'analyse de la variance a permis, à partir de variables caractéristiques des différentes échelles, d'expliquer respectivement $51,1 \%$ et $69,8 \%$ de la variabilité des taux d'occupation de l'habitat physique par les densités et les biomasses totales de truite. Les résultats sont discutés en terme d'apport d'une approche multi-échelles dans la définition du concept de capacité d'accueil d'un cours d'eau à Salmonidés, et de relation entre processus biologiques et échelles d'hétérogénéité de l'habitat.

Mots-clés : truite commune, Salmo trutta, habitat, échelles spatiales, microhabitats.

\section{MULTI-SCALES APPROACH OF THE RELATIONSHIPS BETWEEN BROWN TROUT (SALMO TRUTTA L.) POPULATIONS AND HABITAT FEATURES IN THE CENTRAL PYRENEES.}

\begin{abstract}
The influence of spatial scales, of reach, of sequence and morphodynamic units on occupation by trout populations of the physical habitat calculated by IFIM methodology was studied on 264 morphodynamic units of 15 streams of the Central Pyrenees (France). A variance analysis model explained respectively $51.1 \%$ and $69.8 \%$ of the variability of the occupation of physical habitat by total density and by total biomass of trout. The results are discussed in relation to the interest of multi-scales approach for the carrying capacity concept of salmonid streams and the relation between biological processes and spatial scales.
\end{abstract}

Key-words : brown trout, Salmo trutta, habitat, spatial scales, microhabitat, IFIM (PHABSIM).

\section{INTRODUCTION}

La variabilité des conditions d'habitat est un élément clé vis-à-vis du fonctionnement des populations et des peuplements (SOUTHWOOD, 1977). Dans le cas des écosystèmes aquatiques, cette variabilité environnementale est placée sous le contrôle des échelles d'espace et de temps (DECAMPS et IZARD, 1992). On doit alors associer à chaque processus biologique, une échelle d'hétérogénéité de l'habitat (POWELL, 1989). Au niveau 
des populations de Salmonidés, l'analyse de la variabilité spatiale a souvent été conduite à une échelle locale ou en intégrant dans une même procédure de régression multiple, des facteurs dont l'échelle de variabilité diffère (FAUSCH et al., 1988). Cette approche analytique limite les perspectives d'interprétation écologique des phénomènes. En effet, la caractérisation de l'habitat trutticole fait appel à des facteurs différents selon l'échelle spatiale choisie (HAURY et al., 1991). La stratégie de recrutement (migration en amont et dans les affluents ou utilisation sur une même séquence de différents faciès pour la reproduction, le grossissement des alevins et des adultes) doit également guider le choix des échelles d'approche des populations de truite dans un bassin hydrographique.

La décomposition de la variabilité des abondances de poissons selon plusieurs échelles spatiales peut apporter de nombreux éléments d'interprétation (MAHON, 1984 ; POIZAT, 1993). Chaque étude en écologie se doit de rechercher l'échelle ou la combinaison d'échelles permettant de maximiser la variabilité spatiale d'un phénomène (MEENTEMEYER, 1990, in BAUDRY, 1992). Etant donnée l'importance des facteurs physiques sur la répartition des organismes dans les milieux aquatiques (FRISSELL et al., 1986) et plus spécialement sur les Salmonidés (LEWIS, 1969), l'influence des échelles l'hétérogénéité de l'habitat sur cette répartition doit être testée. La méthode des microhabitats (BOVEE, 1982 ; SOUCHON et al., 1989) permet de définir une capacité d'accueil physique potentielle vis-à-vis de différents stades de développement de la truite commune (Salmo trutta L.). L'hypothèse, selon laquelle la variabilité du taux d'occupation de cet habitat physique potentiel par les truites cumule les effets de processus relatifs à des échelles supérieures ou à d'autres variables de la même échelle, a été testée.

\section{MATÉRIELS ET MÉTHODES}

Les échantillonnages ont été effectués selon un plan complet d'observation respectant trois échelles spatiales : le tronçon ou secteur écoiogique, la séquence et le faciès. Le choix des sites d'études a été guidé par la nécessité de couvrir une gamme de situations contrastées tant du point de vue morphodynamique que physico-chimique.

Le tronçon correspond aux grandes unités hydrodynamiques. Trois types ont été différenciés selon les critères et la classification proposés par BRAVARD et GILVEAR (1993). Cette identification s'est appuyée sur la forme du plancher alluvial et de la vallée, ainsi que sur la pente du cours d'eau. Successivement, chaque rivière a été divisée en tronçons de type gorge (plancher alluvial étroit, forte pente du cours de la rivière et des versants), montagnard (plancher alluvial étroit, pentes moyennes) et plateaux (plancher alluvial large, pentes faibles). Le type plateau regroupe des tronçons à méandres et à tresses (BRAVARD et GILVEAR,1993). La longueur des secteurs varient de 3 à $11 \mathrm{~km}$.

La séquence correspond à une succession de faciès d'écoulement (MALAVOI, 1989). C'est l'échelle de la station d'étude (FRISSELL et al., 1986). Elle a été caractérisée par les différents types de faciès d'écoulement qui la composait, leur diversité (Indice de Shannon) mais également par les conditions physico-chimiques (température, conductivité et nitrates). Nous avons considéré que dans les rivières pyrénéennes, la variabilité de la qualité de l'eau s'exprimait à l'échelle de la séquence plutôt qu'à celle du tronçon. En effet, de par leur longueur importante (souvent supérieure à $5 \mathrm{~km}$ ), chaque tronçon ne peut être caractérisé par des conditions physico-chimiques homogènes.

Le faciès d'écoulement correspond à une zone où les caractéristiques morphodynamiques (pente, vitesse et profondeur) sont homogènes (MALAVOI, 1989).

Deux cent soixante-quatre faciès appartenant à 72 stations (altitudes variant entre 580 et $2050 \mathrm{~m}$, largeurs entre 3 et $12 \mathrm{~m}$ et modules entre 0,2 et $4,4 \mathrm{~m}^{3} / \mathrm{s}$ ) de 15 rivières des Pyrénées centrales ont été échantillonnés en période de débit d'étiage hivernal. En effet, selon l'hypothèse de BOVEE (1982), à l'exception des alevins, l'étiage constitue potentiellement une période d'habitat limitant pour les Salmonidés. Sur la majorité des sites, la truite commune (Salmo trutta L.) est la seule espèce présente, accompagnée quelquefois du chabot (Cottus gobio L.) ( 9 sites). Les quinze rivières étudiées possèdent toutes des régimes hydrologiques de type nivaux. II est important de signaler que 49 sites ont un débit réduit par des installations hydroélectriques. 
Pour chaque faciès d'écoulement, les populations de truites ont été échantillonnées par pêche électrique en utilisant la méthode des passages successifs (2 passages) de DE LURY (DE LURY, 1951).

De même sur chaque unité, à partir des valeurs de vitesse, de profondeur et de substrat mesurées le long de transects transversaux, le modèle biologique PHABSIM de la méthode des microhabitats (BOVEE, 1982) a permis de calculer la Surface Pondérée Utile (SPU) et la Valeur d'habitat (Vh) pour chaque stade de développement de la truite. Les courbes de préférences utilisées sont celles fournies pour les rivières des Pyrénées et du Sud-Ouest par BELAUD et al. (1989). En rapportant les biomasses et densités totales de truite à la surface d'habitat physique favorable dans le faciès à l'étiage (SPUe), il est possible de calculer le taux d'occupation de cet habitat physique potentiel $(T)$ :

$$
\mathrm{T}=\text { biomasse ou densité ( } \mathrm{g} \text { ou ind.)/S.P.U.e }\left(\mathrm{m}^{2}\right) \text {. }
$$

Les surfaces totales d'abris (sous-berges, sous-blocs, branchages et zones profondes) mesurées selon BINNS (1982) ont complété les mesures d'habitat physique.

Dans chaque station, les caractéristiques physico-chimiques (température, conductivité et nitrates) ont été mesurées.

\section{TRAITEMENT STATISTIQUE}

Le traitement statistique a visé à décomposer la variabilité des taux d'occupation de l'habitat physique selon chaque niveau du plan d'observation (les échelles d'espace). La décomposition a été effectuée des échelles supérieures (tronçon) vers les échelles inférieures (faciès). Ce modèle de décomposition successive de la variance (POIZAT, 1993) a reposé, selon le type de variable explicative utilisée (discrète ou continue), sur une analyse de la variance ou sur une régression multiple progressive. Au préalable, il a été nécessaire de s'assurer que l'effet d'une variable à une échelle donnée n'était pas lié à celui d'une variable intervenant à une échelle inférieure. Pour cela, l'homogénéité, entre chaque modalité des variables de niveau supérieur, des distributions des fréquences de chaque facteur, a été testée (test de Kolmogorov-Smirnov (SPRENT, 1992)).

La variabilité totale $(V)$ des taux d'occupation a donc été décomposée :

$$
V=V(T r)+V(S q / T r)+V(S q . T r)+V[f a c /(S q / T r)]+\text { Res }
$$

avec $V(T r):$ effet tronçon,

$\mathrm{V}(\mathrm{Sq} / \mathrm{Tr})$ : effet des variables de la séquence sachant le tronçon,

$\mathrm{V}[\mathrm{fac} /(\mathrm{Sq} / \mathrm{Tr})]$ : effet des variables du faciès sachant la séquence et le tronçon,

Res : le résidu.

La variabilité totale expliquée ( $V$ ) est donc égale à la somme des variances expliquées par les combinaisons de variables de chaque échelle spatiale. En l'absence de normalité des données, des tests par permutations ont permis d'estimer la signification des effets observés (pourcentages de variabilité expliquée) (SPRENT, 1992 ; POIZAT, 1993). Le risque $p$ correspond sous l'hypothèse $(\mathrm{Ho})$ d'absence d'effet du facteur étudié, au rapport du nombre de valeurs obtenues supérieures à la valeur observée sur le nombre de permutations effectuées $(N)$. Ce risque a été estimé avec une précision de $\pm 1.96 \times \sqrt{ } \mathrm{p}(1-\mathrm{p}) / \mathrm{N}(\mathrm{POIZAT}, 1993)$.

\section{RÉSULTATS}

\section{Analyse à l'échelle du faciès seul}

Les valeurs d'habitat calculées pour un débit d'étiage sont faiblement corrélées aux biomasses et densités totales de truite. Pour les biomasses, on observe les plus fortes 
corrélations avec les valeurs d'habitat calculées pour les adultes $\left(r^{2}=0.18, p<0.05\right)$, tandis que les densités sont plus fortement corrélées avec les valeurs d'habitat calculées pour les juvéniles $\left(r^{2}=0.073, p<0.05\right)$. Les taux d'occupation ont donc été calculés en ramenant les biomasses aux SPU des adultes et les densités aux SPU des juvéniles.

\section{Effet tronçon}

L'appartenance à un type de tronçon explique $0,7 \%(p<0.05)$ de la variabilité des taux d'occupation par les densités totales de truite et $5 \%(p<0.05)$ pour les taux d'occupation par les biomasses (Tableau I).

\section{Tableau I : Moyennes des taux d'occupation de la SPU par type de tronçon.}

Table I : Means of occupation of WUA for the three types of reach.

\begin{tabular}{lcc}
\hline $\begin{array}{c}\text { Type de } \\
\text { tronçon }\end{array}$ & $\begin{array}{c}\text { Taux d'occupation } \\
\text { de la SPU étiage } \\
\text { par les densités } \\
\text { (ind. } / \mathrm{m}^{2} \text { de SPUe) }\end{array}$ & $\begin{array}{c}\text { Taux d'occupation } \\
\text { de la SPU étiage } \\
\text { par les biomasses } \\
\left(\mathrm{g} / \mathrm{m}^{2} \text { de SPUe) }\right.\end{array}$ \\
\hline Plateau & 1.44 & 43.4 \\
Montagnard & 1.50 & 59.7 \\
Gorge & 1.17 & 43.8 \\
\hline
\end{tabular}

\section{Effet séquence}

La combinaison des variables concentration en nitrates, conductivité et température explique de $22,5 \%(p<0.05)$ de la variabilité des résidus des taux d'occupation par les densités issus de l'analyse au niveau du tronçon à $23,7 \%(p<0.05)$ pour les résidus des taux d'occupation par les biomasses. Les résidus augmentent avec les valeurs des trois paramètres et ceci dans la gamme de variation étudiée (température de 9 à $13,5^{\circ} \mathrm{C}$, conductivité de 40 à $210 \mu \mathrm{S} / \mathrm{cm}$ et nitrates de 0,4 à $2,5 \mathrm{mg} / \mathrm{l})$.

Quatre variables d'habitat physique de la séquence ont été sélectionnées par la procédure de régression multiple progressive : le pourcentage de faciès radier, de faciès rapide, la diversité des faciès, et la quantité d'habitat favorable aux adultes dans la séquence exprimée en terme de valeur d'habitat. Elles expliquent de $23,8 \%(p<0.05)$ à $21,5 \%(p<0.05)$ de la variablité des résidus des combinaisons tronçon $x$ physico-chimie.

La présence du chabot dans la station n'influence pas en revanche les résidus dans le cas des 9 stations où les 2 espèces cohabitent.

\section{Effet faciès}

La combinaison des variables d'abris explique $4,1 \%(p<0.05)$ de la variablité des résidus issus de l'analyse tronçon $x$ séquence pour les taux d'occupation par les densités et $19,6 \%(p<0.05)$ pour les taux d'occupation par les biomasses. Dans les deux cas, l'habitat physique potentiel du faciès est d'autant plus fortement occupé que la quantité d'abris dans le faciès augmente.

L'intégration de variables d'habitat intervenant à différentes échelles spatiales permet d'expliquer une part importante de la variabilité du taux d'occupation de l'habitat physique potentiel d'un faciès en débit d'étiage par les populations de truites $(51,1 \%$ pour les taux d'occupation par les densités et $69,8 \%$ pour les biomasses - Tableau II). 
Tableau II : Pourcentages de variabilité des taux d'occupation expliqués par les différentes échelles d'espace.

Table II : Percentages of the variability of the occupation of WUA explained by the different spatial scales.

\begin{tabular}{lcc}
\hline & $\begin{array}{c}\text { Taux d'occupation } \\
\text { de la SPU étiage } \\
\text { par les densités }\end{array}$ & $\begin{array}{c}\text { Taux d'occupation } \\
\text { de la SPU étiage } \\
\text { par les biomasses }\end{array}$ \\
\hline Tronçon & 0.7 & 5.0 \\
Séquence & 46.3 & 45.2 \\
Faciès & 4.1. & 19.6 \\
\hline Total & 51.1 & 69.8 \\
\hline
\end{tabular}

\section{DISCUSSION}

En écologie des Salmonidés, de nombreuses études à l'échelle locale relient les caractéristiques de l'habitat à l'abondance des poissons en utilisant très souvent les abris (LEWIS, 1969 ; WESCHE et al., 1987 ; BARAN et al., 1993) ou la valeur d'habitat calculée par la méthode des microhabitats (FAUSCH et al., 1988 ; SOUCHON et al., 1989 ; JOWETT, 1992). A l'opposé, certains travaux montrent les limites de ces approches locales en terme de relation abondances-habitat (SHIRVELL et MORANTZ, 1983 ; BOWLBY et ROOF, 1986 ; CONDER et ANNEAR, 1987 ; PLATTS et NELSON, 1988). Cette absence de relations peut être liée soit à l'influence d'autres paramètres du milieu non utilisés dans l'analyse (BOWLBY et ROOF, 1986 ; SHIRVELL, 1986), soit à la variabilité temporelle (PLATTS et NELSON, 1988). NEWCOMBE (1981) ou encore ORTH (1987) précisent que la qualité de l'habitat physique estimée par la méthode des microhabitats ne constitue qu'une partie seulement de la capacité d'accueil globale. Les abondances de poissons seront dépendantes d'autres variables (température, ressources trophiques, prédateurs...). La majorité des études abordant l'intégration de facteurs de nature différente dans l'explication de la variabilité des abondances, utilisent une procédure de régression multiple progressive (FAUSCH et al., 1988). Si cette procédure permet la construction de modèles déterministes, elle ne permet pas en revanche d'envisager de façon distincte l'effet des différents facteurs, limitant ainsi les possibilités d'interprétation écologique des phénomènes (LEGENDRE et LEGENDRE, 1984). En structurant l'analyse en fonction des échelles de variabilité spatiale des différentes caractéristiques de l'environnement, il est possible d'envisager la part respective de chacun des facteurs dans la variabilité globale des abondances. Les travaux sur le concept d'organisation hiérarchique des systèmes écologiques (ALLEN et STARR, 1982, in BAUDRY, 1992), ainsi que sur l'importance des différentes échelles spatiales (MORRIS, 1987 ; WIENS, 1989 ; POIZAT, 1993), mettent en évidence l'insuffisance des approches locales en terme de biologie des populations. En effet, les échelles spatiales sont des éléments clés dans la structuration des hydrosystèmes (AMOROS et PETTS, 1993). Pour les Salmonidés, KOZEL et al. (1990) suggèrent une étude séparée des relations avec l'habitat physique en fonction de la pente du secteur. MILNER et al. (1985) insistent sur l'importance des caractéristiques du bassin versant, du climat et de la géomorphologie dans le contrôle des conditions locales d'habitat et donc des populations de Salmonidés. JOWETT (1992) explique $82 \%$ de la variabilité d'abondance des truites de taille supérieure à $200 \mathrm{~mm}$ en utilisant des variables hydrologiques, d'habitat physique et du bassin versant. II signale toutefois que les mécanismes par lesquels les caractéristiques du bassin versant agissent sur les abondances de truites sont difficiles à connaître et pose ainsi le problème de la relation entre effet et causalité.

Pour les Pyrénées centrales, les différentes échelles d'espace et surtout les variables qui y sont rattachées, ont un effet significatif sur l'occupation de l'habitat physique. L'effet du tronçon doit être analysé du point de vue de l'influence de la pente et de la forme du plancher alluvial. Les fortes pentes et notamment la présence de barrières infranchissables constituent 
des facteurs limitants à l'occupation de l'habitat physique (LENON, 1967, in ENSIGN et STRANGE, 1990). La limitation des processus de migration et de dévalaison est un élément défavorable à une utilisation optimale de l'espace par les Salmonidés (NORTHCOTE, 1992). Bien que, dans les rivières pyrénéennes, on ne retrouve pas de ségrégation spatiale amontaval au niveau des habitats de reproduction, de grossissement des alevins ou des adultes (BARAN et al., 1993), l'effet du type de tronçon indique que les processus liés aux déplacements des poissons participent à une occupation optimale de l'habitat physique d'un faciès d'écoulement. Des discontinuités entre les habitats constituent des éléments défavorables aux populations de truites. II semble également pertinent d'analyser l'effet tronçon en considérant leur comportement vis-à-vis des événements hydrologiques. En effet, les plateaux dont le substrat est essentiellement composé de dépôts glaciaires, constituent des milieux instables en période de crues avec un important remaniement des matériaux (BRAVARD et GILVEAR, 1993). De même, les gorges, avec une forte contrainte du lit majeur, peuvent présenter des conditions hydrodynamiques très défavorables au moment des forts débits avec d'importantes variations des flux hydriques (BRAVARD et GILVEAR, 1993) couplées à de faibles potentialités d'abris. NEHRING et MILLER (1987) indiquent d'ailleurs que, sur les rivières du Colorado, ce sont les périodes de crues qui représentent les événements à habitat limitant pour les alevins de truites. Dans les rivières pyrénéennes, au vu des pourcentages de variabilité expliquée par les différentes caractéristiques mesurées, il semble que la période d'étiage hivernal joue un rôle important en terme de conditions d'habitat limitantes, la période d'étiage estival étant du fait des régimes hydrologiques peu ou pas marqué sur la majorité des cours d'eau étudiés. Toutefois, l'évolution des conditions d'habitat lors des périodes de fortes eaux ne doit pas être négligée. L'étiage hivernal ne doit pas être considéré comme la seule période d'habitat critique influençant la construction de la population de truites.

Au niveau des conditions physico-chimiques, on peut relier leur influence en terme de processus biologique, à la croissance des individus (BOWLBY et ROOF, 1986 ; BAGLINIERE et MAISSE, 1990). L'influence des caractéristiques de la séquence souligne quant à elle, l'intérêt de la diversité des faciès vis-à-vis des populations de truites. En terme de processus biologique, la diversité permet une ségrégation spatiale des différentes classes de taille et limite ainsi la compétition intraspécifique (BOLHIN, 1977 ; BAGLINIĖRE et CHAMPIGNEULLE, 1982 ; BISSON et al., 1982).

A l'échelle du faciès, l'influence des abris montre bien la nécessité écologique et la complémentarité entre les microhabitats de nutrition (abordés par la SPU) et les microhabitats de repos ou d'affût (abordés par les surfaces d'abris) dans l'activité biologique des individus.

On peut donc relier la variabilité d'abondances observée à chaque échelle spatiale avec des processus biologiques qui s'exprimeront soit à l'échelle de l'individu (importance de la quantité de microhabitats et d'abris, des conditions physico-chimiques), soit à l'échelle de la population (importance de la diversité des faciès et des conditions de circulation).

De plus, les processus de contrôle des caractéristiques propres à chaque échelle d'espace possèdent leur propre vitesse de fonctionnement. Ainsi, la différenciation des tronçons dépendra de processus agissant à l'échelle des temps géologiques (alternance des périodes glaciaires et inter-glaciaires), tandis que l'hétérogénéité d'habitat de la séquence sera influencée par les effets des cycles hydrologiques (crues).

Les caractéristiques propres à chaque échelle d'espace doivent donc être intégrées comme élément de la capacité d'accueil d'un tronçon de rivière vis-à-vis d'une population de truite. La décomposition de la variabilité d'un phénomène biologique par analyse de la variance en utilisant les différentes échelles d'espace comme niveau d'analyse, constitue une démarche pertinente du point de vue de l'interprétation écologique de ces phénomènes. Pour les populations de truites des Pyrénées centrales, cette démarche analytique a permis de constater que les processus relatifs à des échelles locales (sélection des faciès) étaient placés sous la contrainte de processus relatifs à des échelles plus larges. Les approches multi-échelles constituent des bases indispensables à la modélisation en écologie (AUGER, 1992). Elles doivent être intégrées à la fois dans la conception de modèles d'habitat à caractère déterministe et dans l'application et l'interprétation des résultats de modèles numériques. 


\section{BIBLIOGRAPHIE}

AMOROS C., PETTS G.E., 1993. Hydrosystèmes fluviaux. Masson, Paris, $300 \mathrm{p}$.

AUGER P., 1992. Quelques outils mathématiques pour l'analyse hiérarchique. In AUGER P., BAUDRY J., FOURNIER F., Hiérarchies et échelles en écologie, 227-242, Naturalia publication, Paris.

BAGLINIĖRE J.L., CHAMPIGNEULLE A., 1982. Densité des populations de truite commune (Salmo trutta L.) et de juvéniles de saumon atlantique (Salmo salar L.) sur le cours principal du Scorff (Bretagne) : préférendums physiques et variations annuelles (1976-1980). Acta Oecol., Oecol. Appl., 3 (3), 241-256.

BAGLINIĖRE J.L., MAISSE G., 1990. La croissance de la truite commune (Salmo trutta L.) sur le bassin du Scorff. Bull. Fr. Pêche Piscic., 318, 89-101.

BARAN P., DELACOSTE M., LASCAUX J.M. et BELAUD A., 1993. Relations entre les caractéristiques de l'habitat et les populations de truites communes (Salmo trutta L.) de la vallée de la Neste d'Aure. Bull. Fr. Pêche Piscic., 331, 321-340.

BAUDRY J.,1992. Introduction générale. In AUGER P., BAUDRY J., FOURNIER F., Hiérarchies et échelles en écologie, 9-18. Naturalia publication, Paris.

BELAUD A., CHAVEROCHE P., LIM P., SABATON C., 1989. Probability of use curves applied to brown trout (Salmo trutta L.) in rivers of Southern France. Reg. Riv. : Res. Manag., Vol 3, 321-336.

BINNS N.A., 1982. Habitat Quality Index : procedure manual. Wyoming Game and Fish Department. $209 \mathrm{p}$.

BISSON P.A., NIELSEN J.L., PALMASON R.A., GROVE L.E., 1982. A system of naming habitat types in small streams, with examples and utilization by salmonids during low streamflow in ARMANTROUT N.B., Acquisition and utilization of aquatic habitat inventory information. 62-73. American Fisheries Society, Western Division, Bethesda, M.D.

BOLHIN T., 1977. Habitat selection and intercohort competition of juvenile sea-trout Salmo trutta. Oikos, 29, 112-117.

BOVEE K.D., 1982. A guide to stream habitat analysis using Instream Flow Incremental Methodology. Instream Flow Information Paper $n^{\circ} 12$, FWS/OBS 82/86, 248 p.

BOWLBY J.N., ROFF J.C., 1986. Trout biomass and habitat relationship in Southern Ontario streams. Trans. Am. Fish. Soc., 115, 503-514.

BRAVARD J.P., GILVEAR D.J., 1993. Structure hydro-géomorphologique des hydrosystèmes. In AMOROS C., PETTS G.E., Hydrosystèmes fluviaux, 83-103, Masson, Paris.

CONDER A.L., ANNEAR T.C., 1987. Test of weighted usable area derived from a PHABSIM model for instream flow studies on trout streams. N. Am. J. Fish. Manag., 7, 339-350.

DECAMPS H., IZARD M., 1992. L'approche multiscalaire des paysages fluviaux. In AUGER P., BAUDRY J., FOURNIER F., Hiérarchies et échelles en écologie, 115126, Naturalia publication, Paris.

DE LURY D.B., 1951. On the planning of experiments for the estimation of fish populations. J. Fish. Res. Bd. Can., 18 (4), 281-307.

ENSIGN W.E., STRANGE R.J., 1990. Summer foods limitation reduces brook and rainbow trout biomass in a southern Appalachian stream. Trans. Am. Fish. Soc., 119, 894-901.

FAUSCH K.D., HAWKES C.L., PARSONS M.G., 1988. Models that predict the standing crop of stream fish from habitat variables. U.S. Forest Service General Technical Report PNW-GTR - 213, 52 p.

FRISSELL C.A., LISS W.J., WARREN C.E., HURLEY M.D., 1986. A hierarchical framework for stream habitat classification : viewing streams in a watershed context. Environ. Manag., 10, 199-214. 
HAURY J., OMBREDANE D., BAGLINIERE J.L., 1991. L'habitat de la truite commune (Salmo trutta L.) en eaux courantes. In BAGLINIERE J.L., MAISSE G., La truite, biologie et écologie, 47-96, INRA Publ., Paris.

JOWETT I.G., 1992. Models of the abundance of large brown trout in New Zealand Rivers. North Am. J. Fish. Manag., 12, 417-432.

KOZEL S.J., HUBERT W.J., PARSONS G.P., 1990. Habitat features and trout abundance relative to gradient in some Wyoming streams. Northwest Science, 63 (4), 175-182

LEGENDRE P., LEGENDRE L., 1984. Ecologie numérique. 1. Traitement multiple des données écologiques. Masson, Paris.

LEWIS S.L., 1969. Physical factors influencing fish populations in pool of a trout stream. Trans. Am. Fish. Soc., 115, 183-195.

MAHON R., 1984. Divergent structure in fish taxocenes of north temperate streams. Can. J. Fish. Aquat. Sci., 41, 330-350.

MALAVOI J.R., 1989. Typologie des faciès d'écoulement ou unités morpho-dynamiques d'un cours d'eau à haute énergie. Bull. Fr. Pêche Piscic., 315, 189-210.

MILNER N.J., HEMSWORTH R.J., JONES B.E., 1985. Habitat evaluations as a fisheries management tool. J. Fish. Biol., 27 (supplement A), 85-108.

MORRIS D.W., 1987. Ecological scale and habitat use. Ecology, 68 (2), 362-369.

NEHRING R.B., MILLER D.D., 1987. The influence of spring discharge levels on rainbow trout and brown trout recruitment and survival, Black Canyon of the Gunnison River, Colorado, as determined by IFIM/PHABSIM models. Proceedings of the Anglers Conference Western Association Fish and Wildlife Agencies, Salt Lake City, Utah.

NEWCOMBE C.P. 1981. A procedure to estimate changes in fish populations caused by changes in stream discharge. Trans. Am. Fish. Soc., 110 (3), 382-390.

NORTHCOTE T.G., 1992. Migration and residency in stream salmonids - some ecological considerations and evolutionary consequences. Nordic Freshw. Res., 67, 5-17.

ORTH D.J., 1987. Ecological considerations in the development and application of instream flow-habitat models. Reg. Riv. : Res. Manag., vol 1, 171-181.

PLATTS W.S., NELSON R.L, 1988. Fluctuations in trout populations and their implications for land-use evaluation. North. Am. J. Fish. Manag., 8, 333-345.

POIZAT G., 1993. Echelle d'observation et variabilité des abondances de juvéniles de poissons dans un secteur aval du Rhône. Thèse, Univ. Lyon I., 217 p.

POWELL T.M., 1989. Physical and biological scales of variability in lakes, estuaries and the coastal ocean. In ROUGHGARDEN J., MAY R.M., LEVIN S.A., Perspectives in ecological theory. 140-156, Princeton University Press, Princeton, New Jersey.

SHIRVELL C.S., 1986. Pitfalls of physical habitat simulation in the Instream Flow Incremental Methodology. Can. Tech. Rep. Fish. Aqua. Sci., 1460, 68 p.

SHIRVELL C.S., MORANTZ D.L., 1983. Assessments of instream flow incremental methodology for Atlantic salmon in Nova Scotia. Trans. Can. Elect. Assoc., vol 22, 83- $\mathrm{H}-108,22 \mathrm{p}$.

SOUCHON Y., TROCHERIE F., FRAGNOUD E., LACOMBE C., 1989. Les modèles numériques des microhabitats des poissons : applications et nouveaux développements. Rev. Sci. Eau., 2, 807-830.

SOUTHWOOD T.R.E., 1977. Habitat, the templet for ecological strategies ? J. Anim. Ecol., 46, 337-365.

SPRENT P., 1992. Pratique des statistiques non-paramétriques. INRA éditions, Paris, $294 \mathrm{p}$.

WESCHE T.A., GOERTLER C.M., FRYE C.B., 1987. Contribution of riparian vegetation to trout cover in small streams. N. Am. J. Fish. Manag., 7, 151-153.

WIENS J.A., 1989. Spatial scaling in ecology. Funct. Ecol., 3, 385-397. 